\title{
Dual- and multi-energy CT: approach to functional imaging
}

\author{
Juergen Fornaro • Sebastian Leschka • Dennis Hibbeln • Anthony Butler • \\ Nigel Anderson • Gregor Pache • Hans Scheffel • Simon Wildermuth • \\ Hatem Alkadhi • Paul Stolzmann
}

Received: 6 July 2010 /Revised: 12 October 2010 /Accepted: 9 December 2010 /Published online: 19 January 2011

(C) European Society of Radiology 2011

\begin{abstract}
The energy spectrum of X-ray photons after passage through an absorber contains information about its elemental composition. Thus, tissue characterisation becomes feasible provided that absorption characteristics can be measured or differentiated. Dual-energy CT uses two $\mathrm{X}$-ray spectra enabling material differentiation by analysing material-dependent photo-electric and Compton effects. Elemental concentrations can thereby be determined using three-material decomposition algorithms. In comparison to dual-energy CT used in clinical practice, recently developed energy-sensitive photon-counting detectors sample the
\end{abstract}

J. Fornaro $\cdot$ S. Leschka $\cdot$ D. Hibbeln $\cdot$ S. Wildermuth

Institute of Radiology, General Hospital,

Saint Gallen, Switzerland

A. Butler $\cdot$ N. Anderson

Centre for Bioengineering, University of Otago,

Christchurch, New Zealand

A. Butler

European Organization for Nuclear Research, (CERN),

Geneva, Switzerland

G. Pache

Department of Diagnostic Radiology,

University Hospital Freiburg,

Freiburg, Germany

H. Scheffel $\cdot$ H. Alkadhi $\cdot$ P. Stolzmann

Institute of Diagnostic and Interventional Radiology,

University Hospital Zurich,

Zurich, Switzerland

H. Scheffel $\cdot$ H. Alkadhi $\cdot$ P. Stolzmann $(\square)$

Department of Radiology, Massachusetts General Hospital,

Harvard Medical School,

Boston, MA 02114, USA

e-mail: pstolzmann@partners.org material-specific attenuation curves at multiple energy levels and within narrow energy bands; the latter allows the detection of element-specific, $k$-edge discontinuities of the photo-electric cross section. Multi-energy CT imaging therefore is able to concurrently identify multiple materials with increased accuracy. These specific data on material distribution provide information beyond morphological CT, and approach functional imaging. This article reviews the principles of dual- and multi-energy CT imaging, hardware approaches and clinical applications.

Keywords Multi-energy · Dual-energy · Computed tomography $\cdot$ Functional imaging $\cdot$ Spectral CT

\section{Introduction}

Multi-energy computed tomography (CT) refers to the use of spectral data allowing differentiation and classification of tissues to obtain material-specific images [1]. This is possible because attenuation of X-ray photons is energydependent and because each substance has a specific attenuation curve.

In dual-energy $\mathrm{CT}$, materials can be differentiated by exposing the tissues to two different $\mathrm{X}$-ray spectra or using a combination detector with two different energy ranges. The analysis technique of these dual-energy data provides information related to the varying response of tissues to Xrays of different energies [2]. Material differentiation and elemental decomposition thus become possible with the application of two different X-ray spectra.

A disadvantage of this technique is that materials with similar attenuation curves cannot be distinguished. Multienergy imaging reveals additional quantitative information on the attenuation curves by employing energy-sensitive, photon- 
counting devices [3, 4]. In addition to dual-energy CT, multienergy CT thereby allows the spectral decomposition of more than one element with a high atomic number (Z-number) [5].

In clinical practice, the introduction of dual-source $\mathrm{CT}$ has re-enlivened dual-energy CT. The simultaneous acquisition of two different spectra has resolved image co-registration problems introduced by temporal changes [6-8]. Thus, changes in contrast enhancement and/or spatial misregistration problems could be solved. Other approaches were introduced through rapid-kV switching or energy-sensitive detectors [9].

Various clinically relevant applications have been established for dual-energy CT based on either the differentiation or quantification of materials. Material differentiation for instance enables the separation of low and high $Z$-number materials, impacting on bone removal post-processing techniques (e.g. differentiation of calcium and iodine) [1012] or urinary stones analysis (e.g. differentiation of uricacid-containing urinary stones and other stone types) [1317]. Material quantification allows for the reconstruction of virtual non-enhanced images in which the iodine content is subtracted from the contrast-enhanced images $[7,8]$. The replacement of non-contrast-enhanced images with virtual non-contrast-enhanced images has shown the capacity to limit radiation to the patient by omitting standard precontrast data acquisitions [7]. Such imaging strategies can be valuably used in vascular $[18,19]$, liver or kidney imaging either by reducing radiation $[20,21]$ or by quantifying iodine uptake $[8,22,23]$. Regarding cardiac imaging, iodine quantification promises to add information about myocardial blood supply to the analysis of the coronary artery morphology $[24,25]$. In pulmonary angiography, obstructive pulmonary diseases [26] and pulmonary embolisms [27] can be delineated, optimally enabling lung perfusion to be assessed. In addition to lung perfusion, quantification of inhaled xenon gas has allowed the accurate assessment of dynamic and static regional ventilation [28].

Data on material distribution and composition provide information beyond or complementary to morphological CT. Thus, we would like to consider dual- and multi-energy imaging as an approach to 'functional imaging'.

This article reviews the principles of dual- and multi-energy CT imaging, hardware approaches and their application.

\section{Basic principles}

The differentiation of tissues in single-energy CT studies is based on voxel-specific CT numbers (and of course morphology). Single-energy CT measures the cumulative attenuation over the entire energy range of energies for all tissues within a voxel, not the response at different, specific energy levels $[1,2$, 6]. The CT number quantified in Hounsfield units is usually displayed in different shades of grey. However, different materials such as calcium and iodine may have similar CT numbers at certain concentrations and therefore will not be differentiated in a single-energy CT acquisition [6].

In general, the attenuation of any material represented by its CT number is basically caused by a combination of photo-electric and Compton effects. These two main mechanisms contributing to $\mathrm{CT}$ attenuation are both energy- and material-dependent. The photoelectric effect
Fig. 1 Material-specific attenuation curves of bone, iodine and water plotted versus photon energy. Note the difference in attenuation between the mean energy of the spectra of $80 \mathrm{kVp}$ and $140 \mathrm{kVp}$ of iodine compared with bone
Total X-ray Attenuation

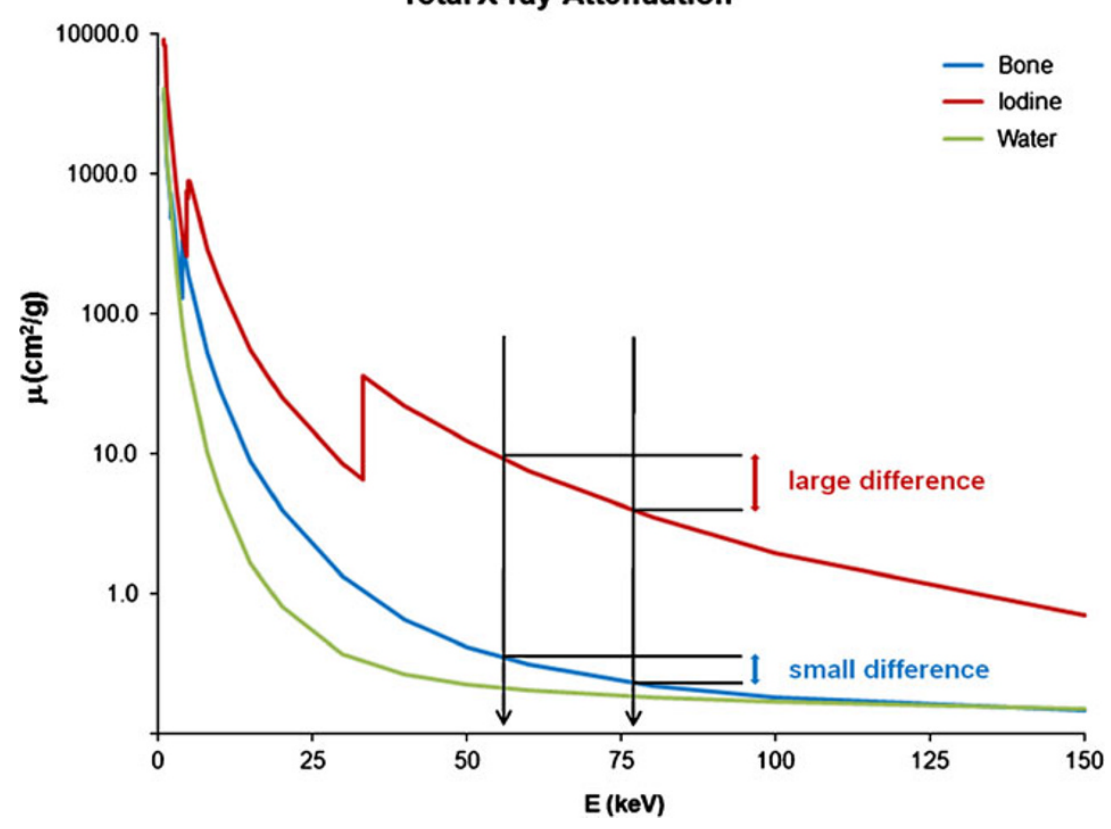


predominates at lower photon energies and is heavily energy-dependent. Compton scattering occurs almost independently of the photon energy at energies exceeding $30 \mathrm{keV}$. The photoelectric effect is related to high atomic numbers, whereas the Compton effect is predominantly related to the density of the material. Therefore, the impact of these two mechanisms varies at different energy levels.

\section{Dual-energy CT}

Computed tomography enables material differentiation by analysing two X-ray spectra, termed dual-energy imaging, allowing the analysis of Compton and photoelectric effects $[1,2]$.

The distinct attenuation values at chosen energy spectra are used for material differentiation in dual-energy $\mathrm{CT}$ imaging provided that the atomic numbers and/or mass densities are different $[1,2]$.

Materials can be distinguished more easily when large differences in atomic numbers are present. Figure 1 illustrates the differentiation of iodine (high atomic number $Z=53$ ) and calcium (atomic number $Z=12-14$ ). Differences in attenuation curves are magnified at two different energy levels, allowing differentiation on the basis of two CT numbers.

Finally, elemental concentrations in dual-energy CT may be determined using three-material decomposition algorithms $[6,7]$. Spectral material decomposition is enabled when the two material-specific CT number measurements of three different materials (i.e. two materials with low $Z$-numbers, e.g. fat and soft tissue $(Z \approx 6-8)$, being different in a singleenergy spectrum, and one material with a high $Z$-number, e.g. iodine $(Z=53)$, being differentiable using dual-energy spectra) are transferred into a scatter-gram plotting CT numbers from the lower energy imaging on the y-axis and $\mathrm{CT}$ numbers from the higher energy imaging on the $\mathrm{x}$-axis. These previously published algorithms then geometrically decompose a single voxel into a mixture of two low $Z$ number elements and the high $Z$-number element (e.g. fat, soft tissue and iodinated contrast material). Assumptions of volume conservation or mass conservation of the voxel are thereby considered, i.e. the sum of the volumes/masses of the three materials should be equal to the volume/mass of the entire voxel [29]. This approach thus not only allows the identification of a single high $Z$-number element, but also its quantification by spectral material decomposition.

\section{Multi-energy CT}

Multi-energy CT is a more specific material decomposition method available for research currently, but not yet available for use in clinical practice. The technique of multi-energy CT differs from that of dual-energy $\mathrm{CT}$ in that it uses the attenuation characteristics at more than two energies (i.e. multiple energies) and within narrow energy ranges [3-5]. The X-ray tube is typically operated at a single tube voltage to produce a broad spectrum of photon energies. The separation of the beam into energy bands happens within the detector, being energy-sensitive photoncounting detectors. An analogy with visible light is that this system is similar to taking a photo of a stained glass window using an ordinary camera. In this system the sun produces a range of wavelengths (or energies); the stained glass window variably attenuates this beam reaching the lens, and the camera's charged-coupled device chip separates the photons into red, green and blue channels.

\section{Hardware approaches}

First attempts to differentiate materials by using the attenuation characteristics at several energy spectra date back to the 1970s [30, 31]. Back in those days, however, technical drawbacks of early CT systems limited the application of dual- and multi-energy CT imaging in daily clinical routine [32].

Despite promising in vitro results, the transferability of results to an in vivo setting was hampered by misregistration problems [33]. In order to obtain two data sets of different Xray energy spectra, acquisitions were performed twice using different peak tube voltages. This approach has carried a significant risk of spatial mismatch ever since. In addition, Xray tube technology did not provide sufficient photon output at low peak voltage to allow diagnostic in vivo imaging at acceptable image noise values [32]. These two major problems - spatial mismatch and noisy images - hindered the transfer of dual-energy CT imaging to clinical radiology; it remained a research technique for many years. However, for projection radiography, dual-energy X-ray absorptiometry had become the reference standard of clinical bone densitometry.

For dual-energy CT, the issues have been resolved by modern CT technology. Technological advances have not only contributed to an improved spatiotemporal resolution, but also significantly shortened the data acquisition time, similarly increasing output power capacity [34]. Currently, CT systems from different vendors are capable of dualenergy material decomposition by evaluating attenuation characteristics of materials at two different energy spectra.

\section{Dual-energy CT}

Three different hardware approaches to obtain dual-energy data have been proposed and pursued by various vendors:

(1) Rapid kVp switching (GE Healthcare, Milwaukee, WI)

(2) Energy-sensitive sandwich detectors (Philips Medical Systems, Cleveland, $\mathrm{OH}$ ) and 


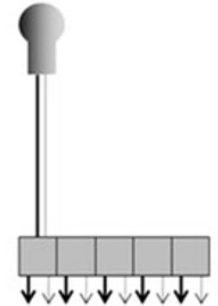

Rapid kVp switching

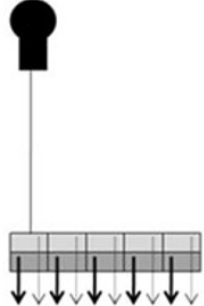

Layer detector

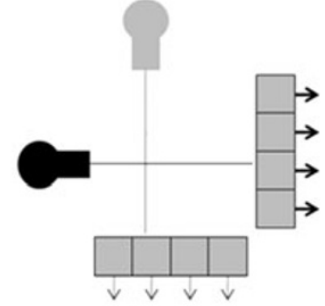

Dual-Source
Fig. 2 Illustration of different hardware approaches to dual-energy $\mathrm{CT}$ imaging. In rapid $\mathrm{kVp}$ switching the $\mathrm{X}$-ray tube voltage is rapidly modulated to different $\mathrm{kVp}$ levels, producing spectra of lower and higher energies (left). Energy-sensitive layer detectors are superimposed on the other (middle). The top-layer detector absorbs lower energy X-ray photons, whereas the bottom-layer detector detects higher energy X-ray photons. The dual-source CT approach uses two $\mathrm{X}$-ray tubes and corresponding detectors arranged at an angular off-set (right). Both X-ray tubes are operated at different $\mathrm{kVp}$ levels, allowing simultaneous dual-energy data acquisition

(3) Dual-source CT (Siemens Healthcare, Forchheim, Germany)

\section{Rapid kVp switching}

In rapid $\mathrm{kVp}$ switching the $\mathrm{X}$-ray tube rapidly modulates the tube voltage, thus producing spectra of lower and higher mean energies as well as peak energies (Fig. 2) [9].

This switching procedure requires doubling the number of projections per rotation to maintain sufficient data for image reconstruction [9].

\section{Energy-sensitive sandwich detectors}

Energy-sensitive sandwich detector technology uses a layer detector design (see Fig. 2). Both detectors are superimposed on the other and are composed of different materials. The toplayer detector absorbs low energy X-ray photons, whereas the bottom-layer detector detects higher-energy X-ray photons [35]. Similar to rapid $\mathrm{kVp}$ switching, the low and high tube voltage output is limited to the same filtration [32].

\section{Dual-source CT}

The third hardware approach is dual-source CT. Dual-source $\mathrm{CT}$ is composed of two X-ray tubes and two detectors arranged at an angular off-set on the rotating gantry (see Fig. 2) [34]. This configuration allows for the evaluation of the coronary arteries at a high temporal resolution [36] or for the acquisition of dual-energy CT data. When both X-ray tubes are operating at different tube voltages, two different $\mathrm{X}$-ray spectra are simultaneously obtained, giving rise to dual-energy tissue characterisation [6,34]. The advantage of the dual-source dual-energy $\mathrm{CT}$ approach is that the tube current can be adjusted for each $\mathrm{kVp}$ level separately, resulting in similar noise levels of the two datasets. Due to the angular off-set of the tube-detector combinations, dualenergy processing has to be performed in the image space.

Current limitations are best understood when considering the following aspect. In general, differentiation of materials with dual-energy CT works especially well when the two energy spectra used have a minor overlap [7]. Because tube voltage set in $\mathrm{CT}$, however, only reflects the peak energy of the X-ray photons, spectral overlap will occur [37]. Finally, because of a small detector size the imaging field of view was limited to the central $26 \mathrm{~cm}$ with first generation dual-source CT hindering imaging of peripheral body parts [8, 26, 28, 34].

These limitations were addressed by second-generation dual-source CT, improving dual-energy imaging capabilities. This CT can be optionally operated with a selective photon shield, represented by a tin filter mounted in front of the highenergy X-ray tube. This filter improves the separation of the two energy spectra (i.e. 80 and $140 \mathrm{kVp}$ ) accompanied by significantly improving discrimination abilities [14, 23, 37].
Fig. 3 Volume rendering of dual-energy cranial CT angiography demonstrates arteriovenous malformation in the right frontal lobe (A, black arrow). Automated bone removal is precisely achieved using dualenergy (B), allowing improved delineation of the malformation together with drainage into the superior sagittal sinus
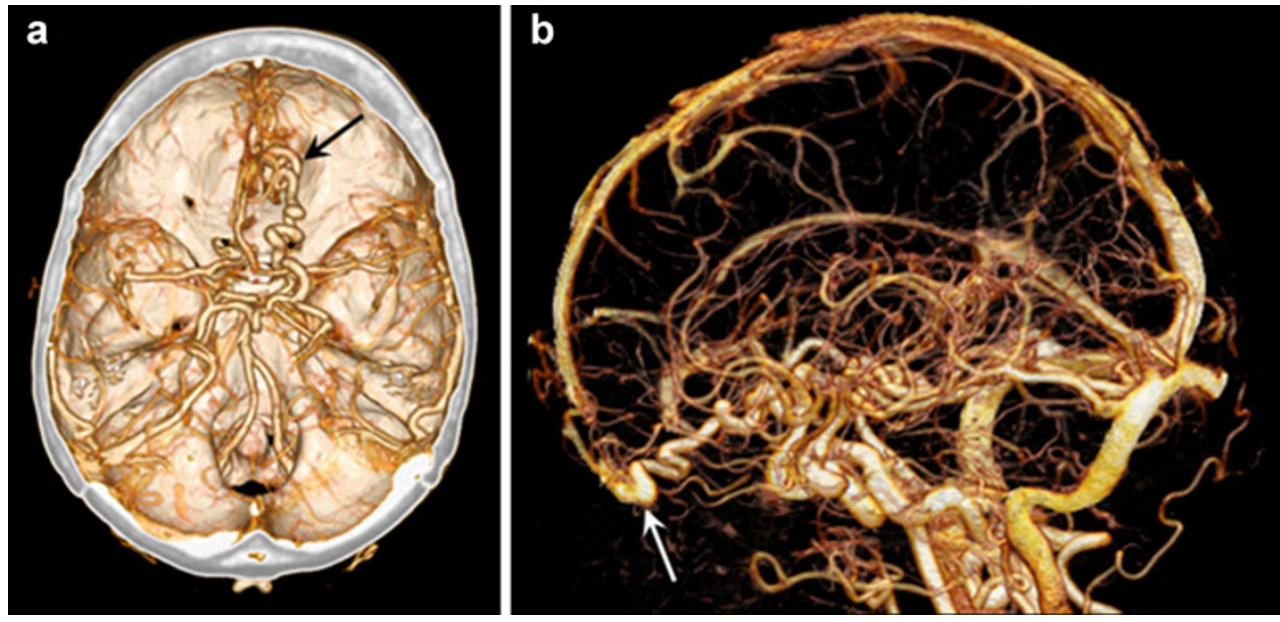
On the other hand, image noise can be lowered significantly when the low $\mathrm{kVp}$ level is increased from 80 to $100 \mathrm{kVp}$ without impacting on discrimination abilities [14]. In order to reduce the radiation dose, investigators evaluated specific low-dose dual-energy protocols as being within the range of single-energy acquisitions [16]. Finally, the imaging field of view was extended to $33 \mathrm{~cm}$, allowing for wider coverage in the $\mathrm{x}$ - and $\mathrm{y}$-directions.

\section{Multi-energy CT}

The latest multi-energy CT systems use photon-counting detectors in order to acquire many X-ray energy levels per image. Such images are often called spectroscopic or spectral CT $[3,38]$. The photon-counting detector technology enables direct conversion of individual X-ray photons into an ionisation cloud within a semi-conductor layer [39]. The detector's electronics then measure the amount of energy deposited by the individual photon and increments a counter associated with that energy. That is, photon counting detectors count individual X-ray photons within set energy ranges - also known as bins or thresholds [3, 4]. Current detectors may have up to eight energy thresholds.

The advantages of CT using energy-sensitive photoncounting detectors are: (1) Each energy is acquired simultaneously so there are no registration problems between different energies. (2) There is no overlap in the energy ranges. (3) Arbitrary energy ranges can be defined to optimise the software in order to discover the imaging problem. (4) As many energy levels as there is room for in the electronics can be recorded.

The Medipix collaboration, hosted by the European Organisation for Nuclear Research (CERN), has been most public with their developments [39], but recently some of the major equipment manufacturers have also announced projects [40].

\section{Imaging applications}

To the best of our knowledge, most recent publications on dual-energy CT have used the dual-source approach.
Fig. 4 Dual-energy CT virtual non-calcium grey-scale (A) and colour-coded images (B) clearly demonstrate post-traumatic bone bruise as proven by $\mathrm{T} 2-$ weighted magnetic resonance imaging $(\mathbf{C})$. Note the intact osseous structures (D)
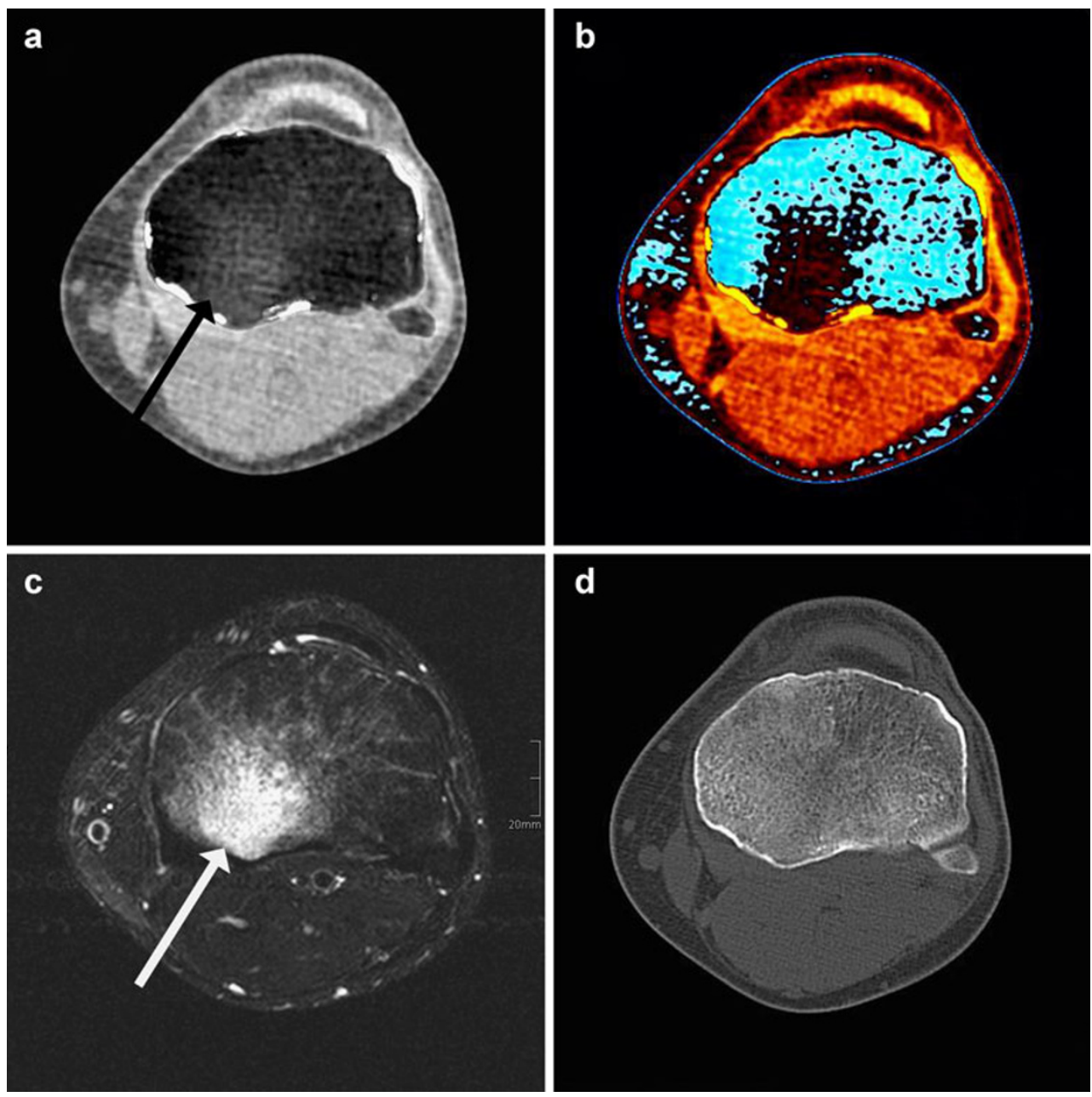


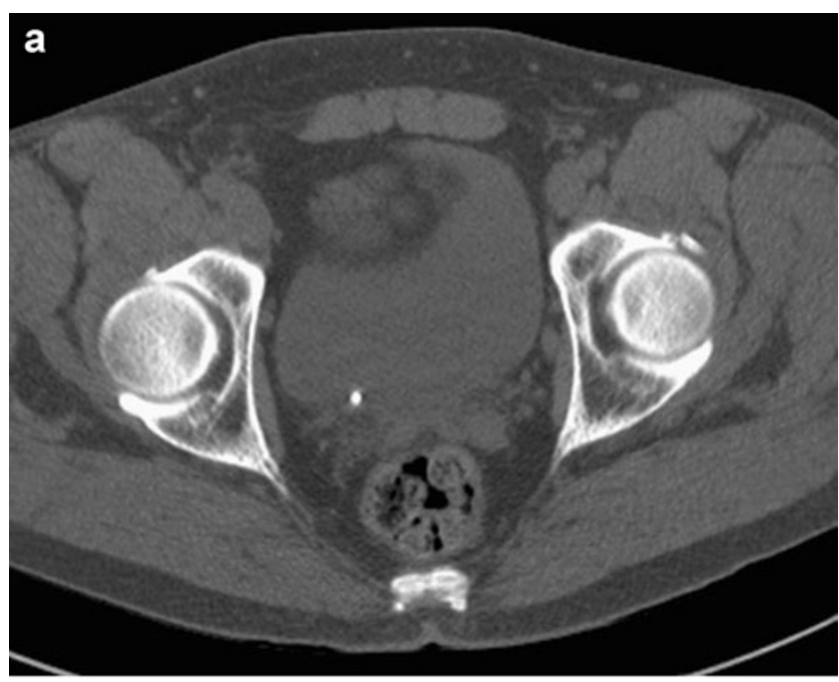

b

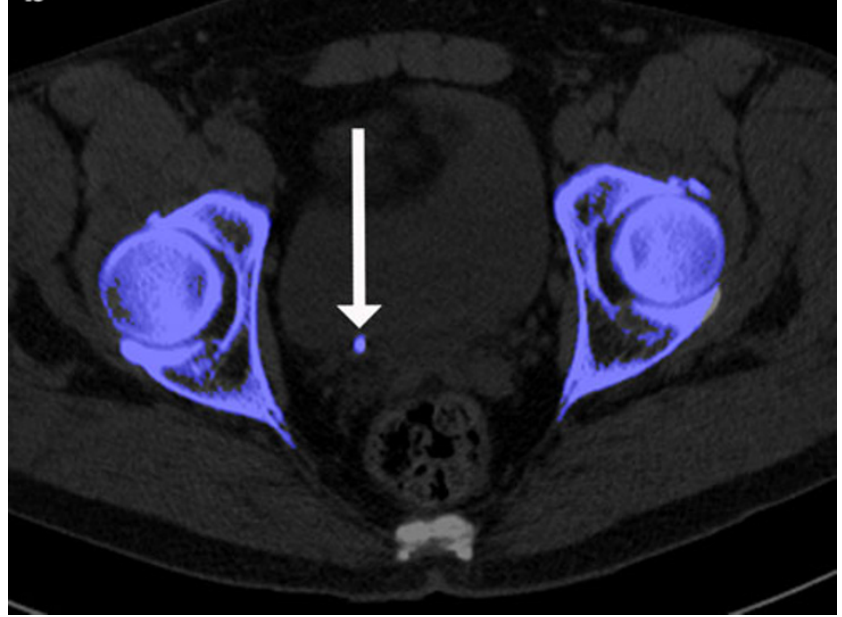

Fig. 5 Non-contrast-enhanced abdominal dual-energy CT accurately depicts a urinary stone in the right distal ureter (A). Dual-energy postprocessing demonstrates the stone to be completely composed of non-uric acid as indicated by blue colour coding (B, arrow). None of the partitions of the stone consists of uric acid (red colour coding, not shown)

A selection of clinically relevant dual-energy CT applications is presented below. Additionally, in order to understand the direction of multi-energy $\mathrm{CT}$, we will take a look at the pre-clinical research laboratories. Applications under development include multi-contrast imaging [3], new contrast agents [41] and atherosclerotic plaque characterisation $[38,42]$.

\section{Dual-energy CT}

Current dual-energy CT applications can be divided into two major groups:

(1) Differentiation of materials

2) Identification and quantification of contrast material
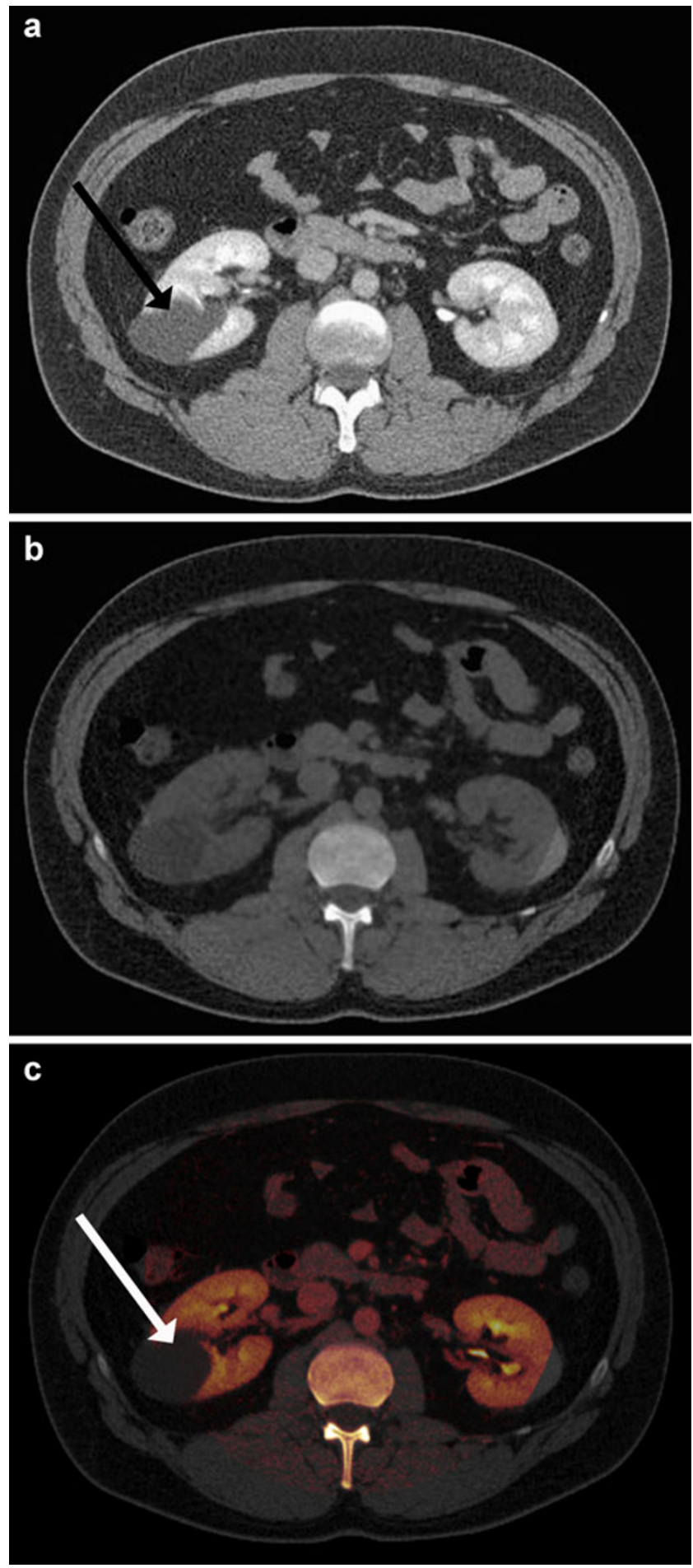

Fig. 6 Weighted dual-energy image (A) averaged between low and high $\mathrm{kVp}$ images similar in appearance to conventional single-energy CT images. Dual-energy-derived material-specific information is used to quantify contrast material, allowing its subtraction to generate virtual non-enhanced images (B). Iodine maps show the contrast material content superimposed on grey scale CT $(\mathbf{C})$. Note comparison of weighted dual-energy image with virtual non-enhanced image as well as iodine maps allowing detection or exclusion of enhancement from a single phase acquisition (see renal cyst, arrows) 

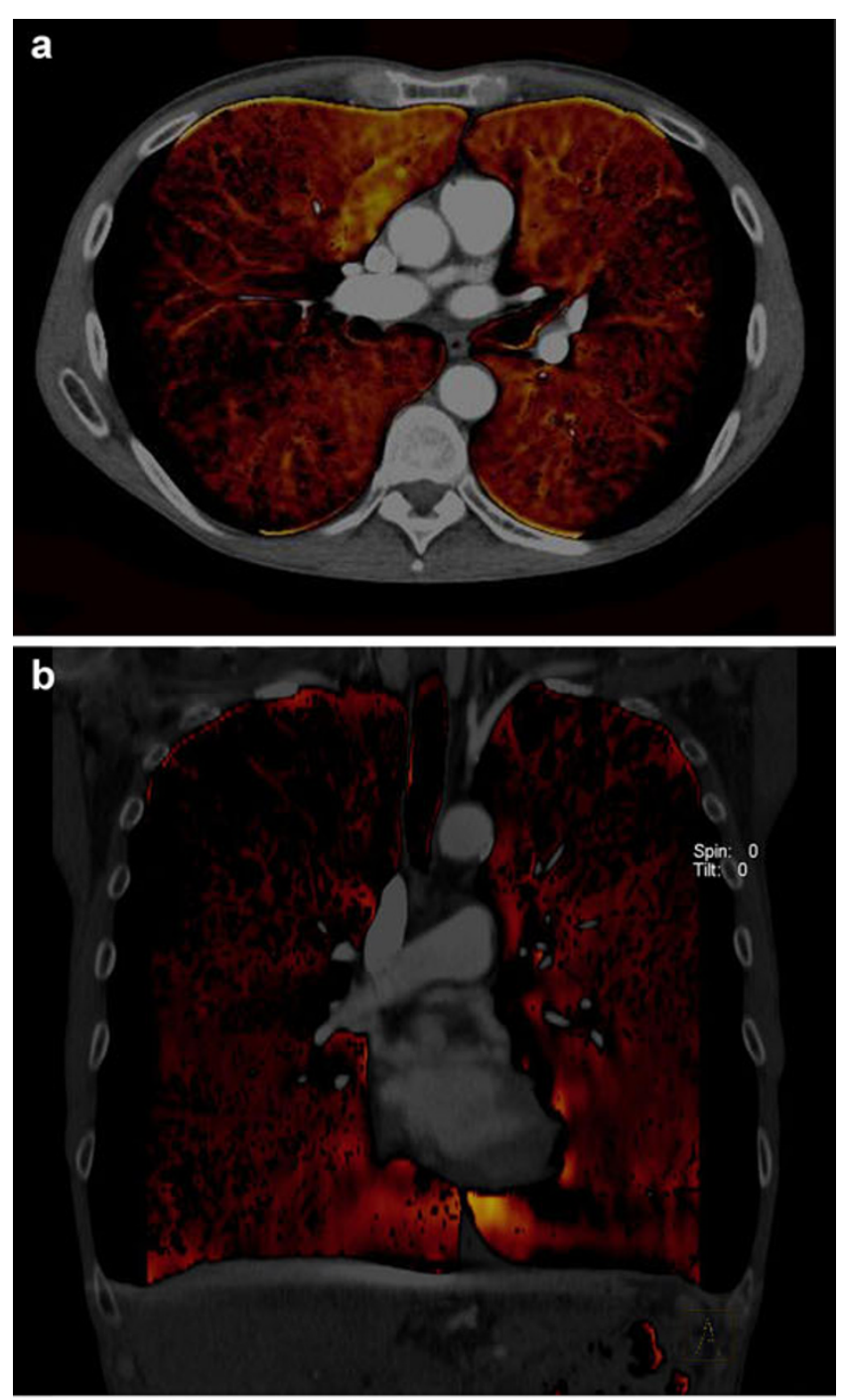

C
Fig. 7 Pulmonary dual-energy CT angiography allows assessment of lung perfusion in patients with pulmonary emphysema. Note the patchy, heterogeneous perfusion pattern mostly accentuated in the right upper lobe as demonstrated by transverse (A) and coronal (B) dual-energy CT reformations being in good agreement with perfusion scintigraphy $(\mathbf{C})$

\section{Differentiation of two materials}

The identification of two different materials basically enables their differentiation (see the above section on Dual-energy CT under Basic principles).

Using material-specific information on calcium and iodine [6, 34], we may not only identify, but also distinguish both in CT angiography. This allows for dual-energy-based postprocessing techniques. Bone removal can be automated on the basis of dual-energy information and need not rely on conventional algorithms (e.g. region growing). This application may be a timeefficient alternative approach in complicated anatomical areas in close proximity to vascular territories and bone such as the circle of Willis and skull (Fig. 3) [11, 43, 44] as well as peripheral arteries and the appendicular skeleton [12, 45].

As bone can precisely be removed from images, bone marrow may become evaluable for CT diagnosis. Most recently, these 'virtual non-calcium images' were evaluated for detecting traumatised bone marrow [46]. Compared with magnetic resonance imaging, dualenergy CT allowed bone marrow assessment, rendering dual-energy CT a potentially valuable tool in the assessment of post-traumatic bone bruises of the knee (Fig. 4).

Not only bone, but also calcified plaques can also be removed from arteries using dual-energy CT. This technique was proven to be applicable in a vessel phantom [47]. The accuracy of this automatic plaque removal was high for calcified plaque, but poorer when low density calcified plaques, low luminal enhancement and small vessel diameters were present. When applied to in vivo vascular imaging, researchers however showed that dual-energy $\mathrm{CT}$ stenosis grading after plaque removal correlated well with digital subtraction angiography in the carotid arteries [10].

According to the same principle used to differentiate calcium (low $Z$-number) from iodine (high $Z$-number), dual-energy CT allows the identification of uric acid. This aids in the workup of urinary stone disease because these uric acid-containing urinary stones can be differentiated from stones of other non-uric acid-containing compositions. This impacts on clinical management because medical treatment may be used to alkalise the urine when uric acid is present. Multiple studies have been performed 
to evaluate this application in vitro $[14,15,48]$ and in vivo $[13,16]$, achieving sensitivities ranging from $88 \%$ to $100 \%$ and specificities ranging from $98 \%$ to $100 \%$ (Fig. 5). Moreover, the transfer of this uric acid identification algorithm to musculoskeletal imaging has been useful in the diagnosis of tophaceous gout [49].

\section{Identification and quantification of contrast material}

One of the major advantages of dual-energy $\mathrm{CT}$ is that the attenuation of iodine increases by a factor of approximately two at $80 \mathrm{kVp}$ compared with $140 \mathrm{kVp}$ [50]. This increased contrast on low $\mathrm{kVp}$ images has proven beneficial to improve the conspicuity of vascularised abdominal organ lesions independently of dualenergy analysis [51].

The contrast material identification contributes not only its quantification (see the above section on Dual-energy CT under Basic principles), but also its subtraction [19-21]. By subtracting iodinated contrast material from the contrastenhanced image, a virtual non-enhanced image is generated (Fig. 6). This application lowers the radiation dose, and non-enhanced acquisitions are subsequently avoided. Virtual non-enhanced images were shown to be an acceptable substitute for standard non-enhanced acquisition regarding renal/adrenal [22, 52] and liver imaging [53] in the followup of endovascular aortic aneurysm repair [18, 19], the detection of urinary stone disease $[20,21]$ and the detection of intracranial haemorrhage in cranial CT angiography [54]. Depending on the protocol used, an up to $50 \%$ reduction in radiation dose is possible when non-enhanced $\mathrm{CT}$ is omitted [7].
On the other hand, the quantification of contrast materials enables the colour-coded display of its elemental iodine concentration (see Fig. 6). This display technique has been investigated for the characterisation of adrenal [22] and renal lesions [52]. In a phantom study assessing renal cysts and enhanced masses, the accuracy for the discrimination was excellent when the iodine overlay technique was used [23]. In a patient study, researchers applied dual-energy CT for characterising renal masses [52] and demonstrated dual-energy $\mathrm{CT}$ after a single nephrographic phase acquisition to correctly identify benign and malignant disease in about $95 \%$ of patients (see Fig. 6).

Dual-energy-derived iodine distribution maps also allowed accurate assessment of cardiac 'perfusion' defects compared with single photon emission CT [24, 25]. Regional alterations of lung perfusion can be depicted by dual-energy $\mathrm{CT}$ in patients suffering from emphysema (Fig. 7) [26]. Similarly, in patients suspected of having pulmonary embolism, several studies have shown that by displaying the iodine distribution in the lung parenchyma, the diagnosis of pulmonary embolism is improved [55].

In addition to the assessment of pulmonary perfusion by iodinated contrast material quantification, dual-energy CT allows the evaluation of pulmonary ventilation by the detection of xenon gas [28]. The detection of xenon gas over time during inhalation facilitates the accurate assessment of dynamic and static regional ventilation. Dualenergy CT thereby significantly reduces the influence of differences in lung volumes during data acquisition compared with single-energy CT. The functional information on ventilation available from dual-energy $\mathrm{CT}$ exactly matches
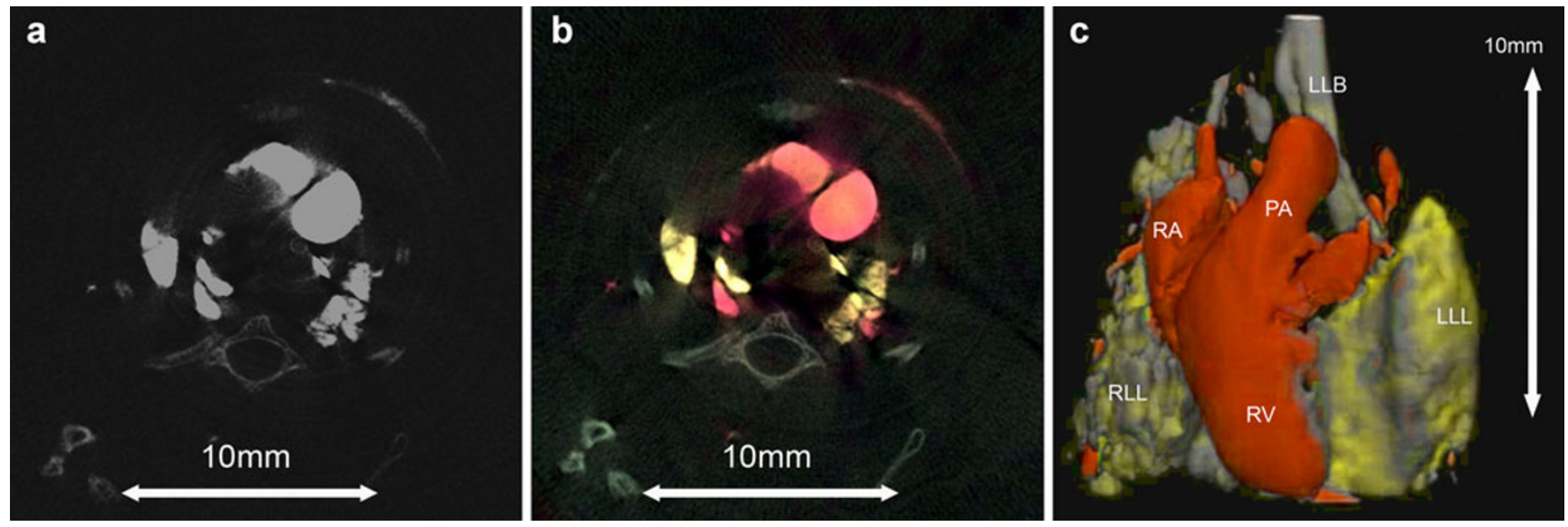

Fig. 8 Multi-energy CT allows differentiation of iodine, barium and bone in mouse. Single-energy image demonstrates similar attenuation values of iodine, barium and bone, which cannot be distinguished on the basis of CT numbers at energies $>23 \mathrm{keV}$ (A). Multi-energy CT data enable differentiation of iodine in circulation (red colour coding), barium in lungs (yellow) and bone (white) on transverse (B) and volume-rendered $\mathrm{CT}$ images $(\mathbf{C})$. Note iodine and barium both have high $Z$-numbers and are solely differentiated on the basis of $k$-edges being $4 \mathrm{keV}$ apart. Left lower bronchus (LLB), left lower lobe (LLL), pulmonary trunk (PA), right atrium (RA), right lower lobe (RLL), right ventricle $(\mathrm{RV})$ 
that from thin-section $\mathrm{CT}$ images generated from the same data set [28].

Measurements of iodine differences might potentially also allow monitoring of tumours after local and antiangiogenesis treatment. However, scientific studies are currently not available; our initial experiences with the detection of the recurrence of hepatocellular carcinoma are promising.

\section{Multi-energy CT}

Pre-clinical applications of multi-energy CT are under investigation in research laboratories.

One group of applications relies on the $k$-edge of multiple contrast agents $[5,40]$. This $k$-edge represents a step change in the attenuation of an element above a particular energy (see Fig. 1). The increased attenuation occurring at a specific energy level of the spectrum corresponds to the binding energy of the $k$-shell electrons above which photoelectric absorption occurs. The ability to identify $k$-edges enables multi-energy $\mathrm{CT}$ to measure any element with a $k$-edge within the diagnostic energy range, allowing multiple high Z-number elements to be distinguished simultaneously [40]. Typically these heavy elements are iodine, barium, gadolinium, gold and platinum.

Multi-contrast $k$-edge imaging has been demonstrated in mice. In particular iodine, barium and background tissues can all be identified as separate materials with mice tissues (Fig. 8) [3]. This implies that multiple radiographic contrast materials could be given at different time points of an examination but imaged at a single phase. In other words, contrast material A could for example be given $15 \mathrm{~min}$ before, and contrast material B $30 \mathrm{~s}$ before a multi-energy $\mathrm{CT}$. Following imaging, using principles similar to virtual non-contrast-enhanced studies, several phases of contrast could be presented to the radiologist, i.e. non-contrast (remove contrast material A and contrast material B), delayed phase (remove contrast material B) and portal venous phase (remove contrast material A). Thus, a triple phase renal or liver study can be performed in a single examination reducing dose, eliminating patient movement and improving patient throughput [3].

The ability to identify heavy elements enables the development of new contrast agents. Many groups have been working on gold-labelled functional nano-particles [41]. Thereby, multi-energy CT imaging enables functional imaging because the gold-labelled particles can be targeted to specific enzymes or cells. Using $k$-edge imaging techniques, it is possible to quantify the amount and location of the particle, even in the presence of other contrast agents such as iodine or gadolinium [5].

Last but not least, multi-energy $\mathrm{CT}$ imaging provides better intrinsic tissue contrast to characterise tissues. An important example is the characterisation of atherosclerotic plaques in which it is desirable to identify iron and calcium to determine the risk of plaque rupture [42].

\section{Conclusions}

Dual- and multi-energy CT improve material differentiation. Different hardware approaches exist for energyselective data analysis and display. Several clinical applications have been established for dual-energy CT imaging, whereas multi-energy CT currently expands on spectral imaging at a preclinical phase. Both dual- and multi-energy $\mathrm{CT}$ emerge as promising techniques providing functional information above and beyond CT imaging of morphology alone.

\section{References}

1. Alvarez RE, Macovski A (1976) Energy-selective reconstructions in X-ray computerized tomography. Phys Med Biol 21:733-744

2. Avrin DE, Macovski A, Zatz LE (1978) Clinical application of Compton and photo-electric reconstruction in computed tomography: preliminary results. Invest Radiol 13:217-222

3. Anderson NG, Butler AP, Scott NJ, Cook NJ, Butzer JS, Schleich N, Firsching M, Grasset R, de Ruiter N, Campbell M, Butler PH (2010) Spectroscopic (multi-energy) CT distinguishes iodine and barium contrast material in MICE. Eur Radiol 20:2126-2134

4. Boll DT, Patil NA, Paulson EK, Merkle EM, Nelson RC, Schindera ST, Roessl E, Martens G, Proksa R, Fleiter TR, Schlomka JP (2009) Focal cystic high-attenuation lesions: characterization in renal phantom by using photon-counting spectral CT-improved differentiation of lesion composition. Radiology 254:270-276

5. Roessl E, Proksa R (2007) K-edge imaging in x-ray computed tomography using multi-bin photon counting detectors. Phys Med Biol 52:4679-4696

6. Johnson TR, Krauss B, Sedlmair M, Grasruck M, Bruder H, Morhard D, Fink C, Weckbach S, Lenhard M, Schmidt B, Flohr T, Reiser MF, Becker CR (2007) Material differentiation by dual energy CT: initial experience. Eur Radiol 17:1510-1517

7. Fletcher JG, Takahashi N, Hartman R, Guimaraes L, Huprich JE, Hough DM, Yu L, McCollough CH (2009) Dual-energy and dualsource CT: is there a role in the abdomen and pelvis? Radiol Clin North Am 47:41-57

8. Graser A, Johnson TR, Chandarana H, Macari M (2009) Dual energy CT: preliminary observations and potential clinical applications in the abdomen. Eur Radiol 19:13-23

9. Kalender WA, Perman WH, Vetter JR, Klotz E (1986) Evaluation of a prototype dual-energy computed tomographic apparatus. I Phantom studies Med Phys 13:334-339

10. Uotani K, Watanabe Y, Higashi M, Nakazawa T, Kono AK, Hori Y, Fukuda T, Kanzaki S, Yamada N, Itoh T, Sugimura K, Naito H (2009) Dual-energy CT head bone and hard plaque removal for quantification of calcified carotid stenosis: utility and comparison with digital subtraction angiography. Eur Radiol 19:2060-2065 
11. Watanabe Y, Uotani K, Nakazawa T, Higashi M, Yamada N, Hori Y, Kanzaki S, Fukuda T, Itoh T, Naito H (2009) Dual-energy direct bone removal $\mathrm{CT}$ angiography for evaluation of intracranial aneurysm or stenosis: comparison with conventional digital subtraction angiography. Eur Radiol 19:1019-1024

12. Yamamoto S, McWilliams J, Arellano C, Marfori W, Cheng W, McNamara T, Quinones-Baldrich WJ, Ruehm SG (2009) Dualenergy CT angiography of pelvic and lower extremity arteries: dual-energy bone subtraction versus manual bone subtraction. Clin Radiol 64:1088-1096

13. Stolzmann P, Kozomara M, Chuck N, Muntener M, Leschka S, Scheffel H, Alkadhi H (2009) In vivo identification of uric acid stones with dual-energy CT: diagnostic performance evaluation in patients. Abdom Imaging 35:629-635

14. Stolzmann P, Leschka S, Scheffel H, Rentsch K, Baumuller S, Desbiolles L, Schmidt B, Marincek B, Alkadhi H (2010) Characterization of urinary stones with dual-energy CT: improved differentiation using a tin filter. Invest Radiol 45:1-6

15. Stolzmann P, Scheffel H, Rentsch K, Schertler T, Frauenfelder T, Leschka S, Sulser T, Marincek B, Alkadhi H (2008) Dual-energy computed tomography for the differentiation of uric acid stones: ex vivo performance evaluation. Urol Res 36:133-138

16. Thomas C, Patschan O, Ketelsen D, Tsiflikas I, Reimann A, Brodoefel H, Buchgeister M, Nagele U, Stenzl A, Claussen C, Kopp A, Heuschmid M, Schlemmer HP (2009) Dual-energy CT for the characterization of urinary calculi: In vitro and in vivo evaluation of a low-dose scanning protocol. Eur Radiol 19:15531559

17. Graser A, Johnson TR, Bader M, Staehler M, Haseke N, Nikolaou K, Reiser MF, Stief CG, Becker CR (2008) Dual energy CT characterization of urinary calculi: initial in vitro and clinical experience. Invest Radiol 43:112-119

18. Chandarana H, Godoy MC, Vlahos I, Graser A, Babb J, Leidecker C, Macari M (2008) Abdominal aorta: evaluation with dual-source dual-energy multidetector CT after endovascular repair of aneurysms-initial observations. Radiology 249:692-700

19. Stolzmann P, Frauenfelder T, Pfammatter T, Peter N, Scheffel H, Lachat M, Schmidt B, Marincek B, Alkadhi H, Schertler T (2008) Endoleaks after endovascular abdominal aortic aneurysm repair: detection with dual-energy dual-source CT. Radiology 249:682691

20. Scheffel H, Stolzmann P, Frauenfelder T, Schertler T, Desbiolles L, Leschka S, Marincek B, Alkadhi H (2007) Dual-energy contrast-enhanced computed tomography for the detection of urinary stone disease. Invest Radiol 42:823-829

21. Takahashi N, Hartman RP, Vrtiska TJ, Kawashima A, Primak AN, Dzyubak OP, Mandrekar JN, Fletcher JG, McCollough CH (2008) Dual-energy CT iodine-subtraction virtual unenhanced technique to detect urinary stones in an iodine-filled collecting system: a phantom study. AJR Am J Roentgenol 190:1169-1173

22. Gupta RT, Ho LM, Marin D, Boll DT, Barnhart HX, Nelson RC (2009) Dual-energy CT for characterization of adrenal nodules: initial experience. AJR Am J Roentgenol 194:1479-1483

23. Leschka S, Stolzmann P, Baumuller S, Scheffel H, Desbiolles L, Schmid B, Marincek B, Alkadhi H (2010) Performance of dualenergy $\mathrm{CT}$ with tin filter technology for the discrimination of renal cysts and enhancing masses. Acad Radiol 17:526-534

24. Ruzsics B, Lee H, Zwerner PL, Gebregziabher M, Costello P, Schoepf UJ (2008) Dual-energy CT of the heart for diagnosing coronary artery stenosis and myocardial ischemia-initial experience. Eur Radiol 18:2414-2424

25. Schwarz F, Ruzsics B, Schoepf UJ, Bastarrika G, Chiaramida SA, Abro JA, Brothers RL, Vogt S, Schmidt B, Costello P, Zwerner PL (2008) Dual-energy CT of the heart-principles and protocols. Eur J Radiol 68:423-433
26. Pansini V, Remy-Jardin M, Faivre JB, Schmidt B, Dejardin-Bothelo A, Perez T, Delannoy V, Duhamel A, Remy J (2009) Assessment of lobar perfusion in smokers according to the presence and severity of emphysema: preliminary experience with dual-energy CT angiography. Eur Radiol 19:2834-2843

27. Thieme SF, Becker CR, Hacker M, Nikolaou K, Reiser MF, Johnson TR (2008) Dual energy CT for the assessment of lung perfusion - correlation to scintigraphy. Eur J Radiol 68:369-374

28. Chae EJ, Seo JB, Goo HW, Kim N, Song KS, Lee SD, Hong SJ, Krauss B (2008) Xenon ventilation CT with a dual-energy technique of dual-source CT: initial experience. Radiology 248:615-624

29. Liu X, Yu L, Primak AN, McCollough CH (2009) Quantitative imaging of element composition and mass fraction using dualenergy CT: three-material decomposition. Med Phys 36:16021609

30. Chiro GD, Brooks RA, Kessler RM, Johnston GS, Jones AE, Herdt JR, Sheridan WT (1979) Tissue signatures with dual-energy computed tomography. Radiology 131:521-523

31. Genant HK, Boyd D (1977) Quantitative bone mineral analysis using dual energy computed tomography. Invest Radiol 12:545551

32. Kelcz F, Joseph PM, Hilal SK (1979) Noise considerations in dual energy CT scanning. Med Phys 6:418-425

33. Grosjean R, Sauer B, Guerra RM, Daudon M, Blum A, Felblinger J, Hubert J (2008) Characterization of human renal stones with MDCT: advantage of dual energy and limitations due to respiratory motion. AJR Am J Roentgenol 190:720-728

34. Flohr TG, McCollough CH, Bruder H, Petersilka M, Gruber K, Suss C, Grasruck M, Stierstorfer K, Krauss B, Raupach R, Primak AN, Kuttner A, Achenbach S, Becker C, Kopp A, Ohnesorge BM (2006) First performance evaluation of a dual-source CT (DSCT) system. Eur Radiol 16:256-268

35. Boll DT, Merkle EM, Paulson EK, Fleiter TR (2008) Coronary stent patency: dual-energy multidetector CT assessment in a pilot study with anthropomorphic phantom. Radiology 247:687-695

36. Alkadhi H, Scheffel H, Desbiolles L, Gaemperli O, Stolzmann P, Plass A, Goerres GW, Luescher TF, Genoni M, Marincek B, Kaufmann PA, Leschka S (2008) Dual-source computed tomography coronary angiography: influence of obesity, calcium load, and heart rate on diagnostic accuracy. Eur Heart J 29:766-776

37. Primak AN, Ramirez Giraldo JC, Liu X, Yu L, McCollough CH (2009) Improved dual-energy material discrimination for dualsource CT by means of additional spectral filtration. Med Phys 36:1359-1369

38. Boll DT, Hoffmann MH, Huber N, Bossert AS, Aschoff AJ, Fleiter TR (2006) Spectral coronary multidetector computed tomography angiography: dual benefit by facilitating plaque characterization and enhancing lumen depiction. J Comput Assist Tomogr 30:804-811

39. Melzer TR, Cook NJ, Butler AP, Watts R, Anderson N, Tipples R, Butler PH (2008) Spectroscopic biomedical imaging with the Medipix2 detector. Australas Phys Eng Sci Med 31:300-306

40. Schlomka JP, Roessl E, Dorscheid R, Dill S, Martens G, Istel T, Baumer C, Herrmann C, Steadman R, Zeitler G, Livne A, Proksa R (2008) Experimental feasibility of multi-energy photoncounting K-edge imaging in pre-clinical computed tomography. Phys Med Biol 53:4031-4047

41. Cormode DP, Skajaa T, Fayad ZA, Mulder WJ (2009) Nanotechnology in medical imaging: probe design and applications. Arterioscler Thromb Vasc Biol 29:992-1000

42. Langheinrich AC, Michniewicz A, Sedding DG, Lai B, Jorgensen SM, Bohle RM, Ritman EL (2007) Quantitative X-ray imaging of intraplaque hemorrhage in aortas of apoE(-/-)/LDL(-/-) double knockout mice. Invest Radiol 42:263-273 
43. Zhang LJ, Wu SY, Niu JB, Zhang ZL, Wang HZ, Zhao YE, Chai X, Zhou CS, Lu GM (2010) Dual-energy CT angiography in the evaluation of intracranial aneurysms: image quality, radiation dose, and comparison with $3 \mathrm{D}$ rotational digital subtraction angiography. AJR Am J Roentgenol 194:23-30

44. Lell MM, Kramer M, Klotz E, Villablanca P, Ruehm SG (2009) Carotid computed tomography angiography with automated bone suppression: a comparative study between dual energy and bone subtraction techniques. Invest Radiol 44:322-328

45. Sommer WH, Johnson TR, Becker CR, Arnoldi E, Kramer H, Reiser MF, Nikolaou K (2009) The value of dual-energy bone removal in maximum intensity projections of lower extremity computed tomography angiography. Invest Radiol 44:285-292

46. Pache G, Krauss B, Strohm P, Saueressig U, Blanke P, Bulla S, Schafer O, Helwig P, Kotter E, Langer M, Baumann T (2010) Dual-energy CT virtual noncalcium technique: detecting posttraumatic bone marrow lesions-feasibility study. Radiology 256:617-624

47. Werncke T, Albrecht T, Wolf KJ, Meyer BC (2010) Dual energy CT of the peripheral arteries: a phantom study to assess the effect of automatic plaque removal on stenosis grading. Rofo 182:682689

48. Primak AN, Fletcher JG, Vrtiska TJ, Dzyubak OP, Lieske JC, Jackson ME, Williams JC Jr, McCollough CH (2007) Noninvasive differentiation of uric acid versus non-uric acid kidney stones using dual-energy CT. Acad Radiol 14:1441-1447
49. Nicolaou S, Yong-Hing CJ, Galea-Soler S, Hou DJ, Louis L, Munk P (2010) Dual-energy CT as a potential new diagnostic tool in the management of gout in the acute setting. AJR Am J Roentgenol 194:1072-1078

50. Schindera ST, Nelson RC, Mukundan S Jr, Paulson EK, Jaffe TA, Miller CM, DeLong DM, Kawaji K, Yoshizumi TT, Samei E (2008) Hypervascular liver tumors: low tube voltage, high tube current multi-detector row CT for enhanced detection-phantom study. Radiology 246:125-132

51. Robinson E, Babb J, Chandarana H, Macari M (2010) Dual source dual energy MDCT: comparison of $80 \mathrm{kVp}$ and weighted average $120 \mathrm{kVp}$ data for conspicuity of hypo-vascular liver metastases. Invest Radiol 45:413-418

52. Graser A, Becker CR, Staehler M, Clevert DA, Macari M, Arndt N, Nikolaou K, Sommer W, Stief C, Reiser MF, Johnson TR (2010) Single-phase dual-energy CT allows for characterization of renal masses as benign or malignant. Invest Radiol 45(7):399-405

53. Zhang LJ, Peng J, Wu SY, Wang ZJ, Wu XS, Zhou CS, Ji XM, Lu GM (2010) Liver virtual non-enhanced CT with dual-source, dualenergy CT: a preliminary study. Eur Radiol 20:2257-2264

54. Ferda J, Novak M, Mirka H, Baxa J, Ferdova E, Bednarova A, Flohr T, Schmidt B, Klotz E, Kreuzberg B (2009) The assessment of intracranial bleeding with virtual unenhanced imaging by means of dual-energy CT angiography. Eur Radiol 19:2518-2522

55. Thieme SF, Johnson TR, Lee C, McWilliams J, Becker CR, Reiser MF, Nikolaou K (2009) Dual-energy CT for the assessment of contrast material distribution in the pulmonary parenchyma. AJR Am J Roentgenol 193:144-149 